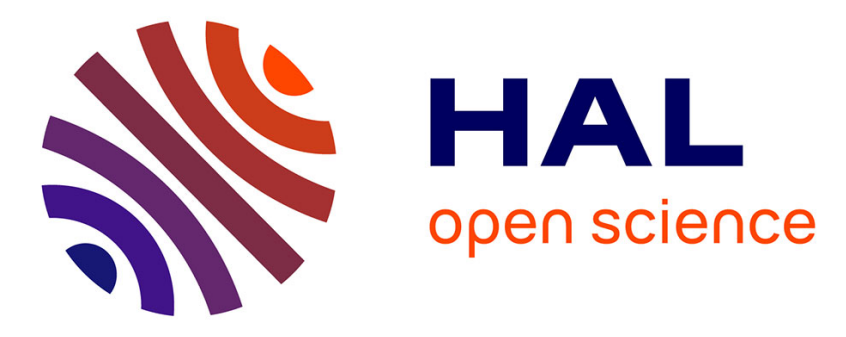

\title{
Phaseless imaging with experimental data: facts and challenges
}

Michele d'Urso, Kamal Belkebir, Lorenzo Crocco, Tommaso Isernia, Amelie Litman

\section{- To cite this version:}

Michele d'Urso, Kamal Belkebir, Lorenzo Crocco, Tommaso Isernia, Amelie Litman. Phaseless imaging with experimental data: facts and challenges. Journal of the Optical Society of America. A Optics, Image Science, and Vision, 2008, 25 (1), pp.271 - 281. 10.1364/JOSAA.25.000271 . hal-00438268

\section{HAL Id: hal-00438268 https://hal.science/hal-00438268}

Submitted on 12 Nov 2018

HAL is a multi-disciplinary open access archive for the deposit and dissemination of scientific research documents, whether they are published or not. The documents may come from teaching and research institutions in France or abroad, or from public or private research centers.
L'archive ouverte pluridisciplinaire HAL, est destinée au dépôt et à la diffusion de documents scientifiques de niveau recherche, publiés ou non, émanant des établissements d'enseignement et de recherche français ou étrangers, des laboratoires publics ou privés. 


\section{Phaseless imaging with experimental data: facts and challenges}

\section{Michele D'Urso}

DIET - Dipartimento di Ingegneria Elettronica e delle Telecomunicazioni, Università degli Studi di Napoli Federico II, Via Claudio 21, I-80125 Naples, Italy

\section{Kamal Belkebir}

Institut Fresnel, UMR-CNRS 6133, Campus de Saint Jérôme, case 162, Université de Provence, F-13397 Marseille Cedex, France

\section{Lorenzo Crocco}

Istituto per il Rilevamento Elettromagnetico dell'Ambiente (IREA) CNR, via Diocleziano 328, I-80124 Naples, Italy

\section{Tommaso Isernia}

DIMET - Università Mediterranea of Reggio Calabria, Loc. Feo di Vito, I-89060 Reggio Calabria, Italy

\section{Amélie Litman}

Institut Fresnel, UMR-CNRS 6133, Campus de Saint Jérôme, case 162, Université de Provence, F-13397 Marseille Cedex, France The aim of this paper is to discuss the characterization of twodimensional targets using inverse profiling approaches with phaseless data. Data correspond to the total fields intensity, which are the actual objects of the measurements devices in many applications. Two different inversion schemes are presented, discussed, compared and validated by using experimental data. In the first one, the intensity-only data are exploited in a minimization scheme, thanks to a proper definition of the cost functional and the evaluation of the associated gradients. The convergence of the iterative 
process takes definite advantage of a suitable normalization and of an useful starting guess which allows us to circumvent the use of a global optimization schemes, which are time consuming. In the second scheme, suggested in [1], one exploits the properties of the scattered fields and the theoretical results on the inversion of quadratic operators to derive a two-steps solution strategy, wherein the (complex) scattered fields embedded in the available data are retrieved first and then a traditional inverse scattering problem is solved. In both cases, the analytical properties and representations of the involved fields allow to properly fix the measurement set-up and to identify the more convenient solution strategy to adopt. Indications on the number and type of primary sources and receivers to be used are also given. Results from experimental data show the efficiency of the above approaches and of the introduced tools.

(C) 2007 Optical Society of America

OCIS codes: 290.3200, 110.6960

\section{Introduction}

In inverse scattering problems, one looks for a quantitatively accurate description of the electrical and geometrical properties of an investigated region given a set of incident fields and measures (both in amplitude and phase) of the corresponding scattered fields on a generic surface lying outside the region under test [2]. Due to their wide range of potential applications, the development of accurate and reliable techniques for solving this kind of problems is nowadays a still important challenge [3-5].

By leaving aside peculiar characteristics of the different approaches proposed in the literature, one of the main common drawbacks resides in the need of measuring both amplitude and phase of the scattered fields. As a matter of fact, in several areas of applied science, the phase distribution of the scattered fields is often too corrupted by noise to be useful, or even there is no phase measurement at all, e.g., optical measurement setup. Even if there is some effort nowadays to provide experimental setups capable of measuring all the components of the scattered fields $[6,7]$, it is of great importance to develop approaches 
that image samples from only amplitude data, as these latter would open the way to more simple and cost effective experimental set-ups. In addition, it is also important to remark that, in most applications, the actual measured quantity is the total field. In fact, unless the incident field is provided by a directive antenna, the measured field in presence of the target contains both the incident and the scattered field, so that the total field has to be processed instead of the scattered field as usually done.

In order to overcome the above limitations, several approaches for solving inverse scattering problems from intensity-only data have been proposed in the literature [1, 8-13]. Among them, an approach based on only amplitude measurements of the total fields has been recently proposed, first with reference to the case of measures taken on a closed curve surrounding the domain under test [1] and then to that of transmitters and receivers placed over two truncated lines somehow enclosing the investigating domain [13]. In both cases, the proposed procedures splits the imaging problem into two different steps. In the first one, the scattered field is estimated from the measures of the square amplitude distributions of the total field, while the second step is aimed at estimating the unknown dielectric properties from the estimated scattered fields (modulus and phase). In summary, the first step allows us to estimate the input data for the second one, which is a traditional inverse scattering problem. Notably, as recalled throughout this paper and in previous contributions [1, 13], the separation of the problem into two different steps allows a better control of the overall non-linearity of the inverse problem with respect to single step procedures. In fact, the exploitation of theoretical results on the inversion of quadratic operators [14] and field properties representations [15, 16], leading to design constraints on the measurement set-up, allows to successfully solve the first step, while all the available knowledge about traditional inverse scattering problems is exploited in the second one. More recently, such an imaging technique has been extended to a three-steps procedure, where a Phase Retrieval (PR) problem is preliminary solved to estimate the phase of the incident field from its measured amplitude [17]. By doing so, the resulting imaging strategy completely relies on only amplitude data.

However, the above mentioned inversion approaches $[1,13,17]$ can be actually applied provided that some conditions on the measurement set-up are satisfied. As a matter of fact, when these conditions do not hold true, the estimation of the scattered field from the measured total field amplitude is not reliable. In these cases, it is therefore of interest to 
develop new, accurate and effective inverse profiling approaches based on only amplitude information of the total field, once known or estimated the incident field in the scattering domain and on the measurement curve. In such approaches, the aim is to solve the imaging problem in a single step, without previously estimating the scattered field embedded in the measures. This would require to reformulate the inverse scattering problem in order to take into account that the available data are intensity only. On the other hand, at least in principle, particular constraints on the measurement set-up are not required. Therefore, these approaches are expected to be useful in all those cases wherein the two-steps strategy $[1,13]$ or its generalization [17] cannot be used.

The aim of this paper is therefore to introduce a novel one-step imaging strategy based on only-amplitude total field data and compare and discuss, by using experimental data, its performances with that of the two-steps strategy.

It is worth noting that the idea of directly incorporating the square amplitude distributions of the total field in the inversion scheme is not new in the literature [10-12]. With respect to these contributions, the approaches proposed and discussed in this paper have interesting and complementary characteristics with respect to the above ones. First, unlike [10], we do not make use of a priori information in the inversion process, but we rather take advantage of a suitable starting guess achieved by means of a simple modification of the widely used backpropagation solution [18]. Moreover, unlike [12], the adopted minimization scheme exploits a local optimization procedure based on an efficient CG-FFT scheme and thus avoids the use of time consuming global optimization algorithms. In this respect, it is also worth noticing that the use of a proper weighting of the cost functional to minimize, derived from the properties of the intensity only data pattern, as well as from the available knowledge in Phase Retrieval procedures [14], allows us to improve the data fitting and of course the final reconstructions in terms of permittivity and conductivity of the unknowns targets. As a last but not least point, let us remark that our approaches are based on the Contrast Source-Extended Born (CS-EB) inversion scheme, which allows to reduce the degree of non-linearity [19] of the inverse scattering problem and achieves improved permittivity and conductivity maps reconstructions in many cases [20].

The paper is organized as follows. In Section 2, the adopted geometry configuration is presented and the mathematical model is given. The sampling properties and representations of the involved electromagnetic fields are also recalled. In Section 3, the single-step 
inversion scheme is thoroughly described, together with the weighting strategy and the adopted modified backpropagation as initial solution. The features and limitations of the two-steps approach are briefly sketched in Section 4. Section 5 is devoted to assess and compare the performances of the two approaches by means of experimental data concerning metallic and dielectric inhomogeneous targets, collected at the Institute Fresnel of Marseille. Conclusions follow.

\section{Mathematical model and field properties}

The geometry of the problem studied in this paper is shown in Fig. 1 where one or more two-dimensional objects of arbitrary cross-section $\Omega$ are confined in a bounded domain $D$. The embedding medium $\Omega_{b}$ is assumed to be infinite and homogeneous, with permittivity $\varepsilon_{b}=\varepsilon_{0} \varepsilon_{b r}$, and permeability $\mu=\mu_{0}\left(\varepsilon_{0}\right.$ and $\mu_{0}$ being the permittivity and permeability of the vacuum, respectively). The scatterers are assumed to be inhomogeneous cylinders with a permittivity distribution $\varepsilon(\mathbf{r})=\varepsilon_{0} \varepsilon_{r}(\mathbf{r})$; the entire configuration is non-magnetic $\left(\mu=\mu_{0}\right)$. A right-handed Cartesian coordinate frame $\left(O, \mathbf{u}_{\mathbf{x}}, \mathbf{u}_{\mathbf{y}}, \mathbf{u}_{\mathbf{z}}\right)$ is defined. The origin $O$ can be either inside or outside the scatterer and the $z$-axis is parallel to the invariance axis of the scatterer. The position vector $\mathbf{O M}$ can then be written as $\mathbf{O M}=\mathbf{r}+z \mathbf{u}_{\mathbf{z}}$. The line sources that generate the electromagnetic excitation (denoted as $T_{x}$ in Fig. 1) and the elementary probes collecting the data $\left(R_{x}\right.$ in Fig. 1) are located at $\left(\mathbf{r}_{l}\right)_{1 \leq l \leq L}$ on a circle $\Gamma$ of radius $R_{\Gamma}$. Taking into account a time factor $\exp (i \omega t)$, in the Transverse Magnetic (TM) case, the time-harmonic incident electric field created by the $l^{\text {th }}$ sources is

$$
\mathbf{E}_{l}^{\mathrm{i}}(\mathbf{r})=E_{l}^{\mathrm{i}}(\mathbf{r}) \mathbf{u}_{\mathbf{z}}=A \frac{\omega \mu_{0}}{4} H_{0}^{(2)}\left(k_{b}\left|\mathbf{r}-\mathbf{r}_{l}\right|\right) \mathbf{u}_{\mathbf{z}}
$$

where $A$ is the strength of the electric source, $\omega$ the angular frequency, $H_{0}^{(2)}$ the Hankel function of zero-order and second kind and $k_{b}$ the wavenumber in the surrounding medium. Under these hypotheses and omitting the $\exp (i \omega t)$ time dependence term, for each illumination condition, the scattering equations describing the total field can be formulated as two coupled contrast-source integral relations [18]: the observation or data equation Eq.( 2) and 
the coupling or state equation Eq.( 3), which are

$$
\begin{aligned}
& E_{l}(\mathbf{r} \in \Gamma)=E_{l}^{\mathrm{i}}(\mathbf{r} \in \Gamma)+E_{l}^{\mathrm{s}}(\mathbf{r} \in \Gamma)=E_{l}^{\mathrm{i}}(\mathbf{r} \in \Gamma)+\iint_{D} G\left(\mathbf{r}, \mathbf{r}^{\prime}\right) J_{l}\left(\mathbf{r}^{\prime}\right), \mathrm{d} \mathbf{r}^{\prime}, \\
& J_{l}(\mathbf{r} \in D)=\chi(\mathbf{r} \in D) E_{l}^{\mathrm{i}}(\mathbf{r} \in D)+\chi(\mathbf{r} \in D) \iint_{D} G\left(\mathbf{r}, \mathbf{r}^{\prime}\right) J_{l}\left(\mathbf{r}^{\prime}\right) \mathrm{d} \mathbf{r}^{\prime},
\end{aligned}
$$

where $\chi(\mathbf{r})=\varepsilon_{r}(\mathbf{r})-\varepsilon_{b r}$ denotes the permittivity contrast which vanishes outside $D$, $G\left(\mathbf{r}, \mathbf{r}^{\prime}\right)$ is the two-dimensional free-space Green function, and $J(\mathbf{r})=\chi(\mathbf{r}) E(\mathbf{r})$ corresponds to the contrast source.

The overall aim of the problem is to determine the two dimensional contrast function $\chi(\mathbf{r})$ in $D$ starting from the knowledge of the incident fields $E_{l}^{\mathrm{i}}(\mathbf{r} \in \Gamma)$ on the probing curve $\Gamma$, and from an incomplete (because only a finite number of measurements can be performed) and inaccurate (because the measurements are error-affected) knowledge of the intensity of the total fields $\left|E_{l}(\mathbf{r} \in \Gamma)\right|^{2}, l \in(1, \ldots, L)$.

To this end, as $|E|^{2}=\left|E^{\mathrm{i}}\right|^{2}+\left|E^{\mathrm{s}}\right|^{2}+2 \Re e\left(E^{\mathrm{s}} E^{\mathrm{i}^{*}}\right)$, it proves fruitful to briefly recall properties and possible representations of both scattered and incident fields, and then of $\left|E^{\mathrm{i}}\right|^{2},\left|E^{\mathrm{s}}\right|^{2}$ as well as of the interference term $\Re e\left(E^{\mathrm{s}} E^{\mathrm{i}^{*}}\right)$. As discussed in the following, these properties will allow to quantify the amount of independent data at our disposal for solving the imaging problem at hand, to sample the intensity data in an accurate and non-redundant fashion and to determine the maximum amount of information about the targets one can extract from the available data. Moreover, as in [1, 13, 17], exploitation of these properties provides the guidelines to design an effective measurement set-up.

With reference to the geometry depicted in Fig. 1, it is known that the scattered field corresponding to a given source can be accurately represented with a finite number of Fourier harmonics given by $2 k_{b} R_{D}, R_{D}$ being the radius of the minimum circle enclosing the targets [16]. As a Fourier series can be turned into a Dirichlet sampling series, $2 k_{b} R_{D}$ samples uniformly spaced in angle accurately represent each scattered field as well. From reciprocity [16], the number of non-superdirective independent incident fields impinging on the domain under test is $2 k_{b} R_{D}$ as well. Hence, by excluding superdirective sources, $2 k_{b} R_{D}$ plane waves uniformly spaced in angle form a complete family of independent incident fields. Therefore, as a function of the incident angle $\vartheta_{l}$ and of the receiving angle $\vartheta_{r}$, the scattered field can be accurately represented by a number of samples given by $\left(2 k_{b} R_{D}\right) \times\left(2 k_{b} R_{D}\right)=\left(2 k_{b} R_{D}\right)^{2}$, where, as discussed in [1], only one half of these samples is actually independent. 
As far as the incident fields measured on $\Gamma$ are concerned, a different result holds true. In fact, by paralleling the above reasoning to the representation of the incident field in $D$, one can prove that each incident field on $\Gamma$ can be accurately represented by $2 k_{b} R_{\Gamma}$ Dirichlet samples, and that $2 k_{b} R_{\Gamma}$ (non-superdirective) independent incident fields (constituted by plane waves uniformly spaced in angle) exist therein. Therefore, as discussed for the scattered field, the incident field on $\Gamma$ as a function of both angles $\vartheta_{l}$ and $\vartheta_{r}$ can be accurately represented by a number of samples given by $\left(2 k_{b} R_{\Gamma}\right) \times\left(2 k_{b} R_{\Gamma}\right)=\left(2 k_{b} R_{\Gamma}\right)^{2}$. Note that, also in this case, only one half of these samples is actually independent [1].

When considering the square amplitude patterns of the above fields, the number of samples required for a faithful representation becomes four times larger (with respect to amplitude and phase measurements) as the sampling step has to be halved along each of the two coordinates. Therefore, $\left|E^{\mathrm{s}}(\mathbf{r} \in \Gamma)\right|^{2}$ requires $\left(4 k_{b} R_{D}\right) \times\left(4 k_{b} R_{D}\right)=\left(4 k_{b} R_{D}\right)^{2}$ samples and $\left|E^{\mathrm{i}}(\mathbf{r} \in \Gamma)\right|^{2}$ requires $\left(4 k_{b} R_{\Gamma}\right) \times\left(4 k_{b} R_{\Gamma}\right)=\left(4 k_{b} R_{\Gamma}\right)^{2}$ samples.

In order to accurately represent $|E|^{2}$ on $\Gamma$, being $|E|^{2}=\left|E^{\mathrm{i}}\right|^{2}+\left|E^{\mathrm{s}}\right|^{2}+2 \Re e\left(E^{\mathrm{s}} E^{\mathrm{i}^{*}}\right)$, one needs a number of samples equal to the maximum between $\left(4 k_{b} R_{\Gamma}\right)^{2}$ and $\left(2 k_{b}\left(R_{D}+R_{\Gamma}\right)\right)^{2}$, the latter being the number of samples required to represent the term $2 \Re e\left(E^{\mathrm{s}} E^{\mathrm{i}^{*}}\right)$ on $\Gamma$ [13]. Of course, only a half of these samples is independent [1].

\section{A single-step approach for intensity only inverse profiling}

Traditionally, in standard inverse scattering problems, one assumes the knowledge of the total fields in both amplitude and phase. Herein, the problem we want to solve consists in retrieving the dielectric characteristics within a region under test from measurements of the square amplitude distribution of the total field, once known (or estimated as in [17]) the incident field. The approach described in this section corresponds to a single-step procedure, based on the minimization of a discrepancy criterion between the amplitude of the simulated and measured total fields. This minimization problem is recast into a Contrast-SourceExtended-Born (CS-EB) formalism as in [20]. A brief recall of derivation and main features of the CS-EB scattering model is reported in the Appendix A. 


\section{A. Unknowns representation}

In the Contrast-Source inversion method [18], both the contrast $\chi$ and the induced current $J=\chi E$ inside the targets are assumed as unknowns. In order to lower the degree of nonlinearity [19] and therefore the difficulty of the inverse problems with respect to parameters embedding dielectric characteristics, the traditional scattering equation Eq.(3) is replaced by a new coupling equation, the Contrast Source - Extended Born (CS-EB) equation [20], given by

$$
J_{l}(\mathbf{r})-\xi(\mathbf{r}) E_{l}^{\mathrm{i}}(\mathbf{r})=\xi(\mathbf{r}) \iint_{D} G\left(\mathbf{r}, \mathbf{r}^{\prime}\right)\left[J_{l}\left(\mathbf{r}^{\prime}\right)-J_{l}(\mathbf{r})\right] \mathrm{d} \mathbf{r}^{\prime}=\xi(\mathbf{r}) \mathbf{G}_{\bmod }\left(J_{l}\right),
$$

where

$$
\begin{gathered}
\xi(\mathbf{r})=\frac{\chi(\mathbf{r})}{1-\chi(\mathbf{r}) f_{D}(\mathbf{r})}, \quad f_{D}(\mathbf{r})=\iint_{D} G\left(\mathbf{r}, \mathbf{r}^{\prime}\right) \mathrm{d} \mathbf{r}^{\prime} \\
\mathbf{G}_{\text {mod }}\left(J_{l}\right)=\iint_{D} G\left(\mathbf{r}, \mathbf{r}^{\prime}\right)\left[J_{l}\left(\mathbf{r}^{\prime}\right)-J_{l}(\mathbf{r})\right] \mathrm{d} \mathbf{r}^{\prime}=\iint_{D} G\left(\mathbf{r}, \mathbf{r}^{\prime}\right) J_{l}\left(\mathbf{r}^{\prime}\right) \mathrm{d} \mathbf{r}^{\prime}-J_{l}(\mathbf{r}) f_{D}(\mathbf{r}) .
\end{gathered}
$$

For the sake of simplicity, equations Eq.(2) and Eq.(4) can be rewritten using symbolic notations as

$$
E_{l}^{\mathrm{s}}=\mathbf{K} J_{l} ; \quad J_{l}=\xi E_{l}^{\mathrm{i}}+\xi \mathbf{G}_{\mathrm{mod}}\left(J_{l}\right),
$$

where $\mathbf{G}_{\text {mod }}\left(J_{l}\right)$ is the new scattering operator relating the induced current inside the scattering domain to the scattered field outside. It is worth noticing that, despite the fact that the CS-EB model defined in Eq.(4) is just a simple rewriting of the traditional contrast source model, it has proved to be a more effective tool to formulate and solve both forward and inverse scattering problems [20]. Notably, while its derivation was inspired by some mathematical and physical considerations related to presence of losses in the host medium and/or in the targets [20], processing of experimental data (both amplitude and phase) has shown that accurate and reliable results can be achieved also for lossless inhomogeneous targets in free space [21].

The ill-posedness of the inverse scattering problem is also dealt with by looking for finitedimensional representations of both the unknowns [22]. We thus consider

$$
\begin{aligned}
& \xi(\mathbf{r})=\sum_{p=1}^{P} a_{p} \psi_{p}(\mathbf{r}) \\
& J_{l}(\mathbf{r})=\sum_{q=1}^{Q} c_{q}^{l} \phi_{q}(\mathbf{r}) \quad \forall l=1, \ldots, L
\end{aligned}
$$


wherein $\left\{\psi_{p}\right\}_{p=1}^{P}$ and $\left\{\phi_{q}\right\}_{q=1}^{Q}$ are two orthonormal basis functions taken here as spatial Fourier harmonics owing to the lack of a priori information on the unknown scatterers. Of course, according to the above results on the field properties, the number of the unknowns coefficients $\left\{a_{p}\right\}_{p=1}^{P}$ and $\left\{c_{q}\right\}_{q=1}^{Q}$ has to be lower than the number of independent data one has at disposal for the inversion as discussed in the previous section (see also [22]). In particular, note that, as far as the choice of $P$ is concerned, the properties of the scattered fields recalled in Section 2 allow to state that, for any given $R_{D}$, one can determine the maximum amount of information which can be extracted in the inverse scattering step, thus allowing to fix the maximum number of unknowns coefficients for the contrast function in Eq.( 7) which can be reliably retrieved.

\section{B. Discrepancy criteria}

The discrepancy criteria between the measured fields and the simulated ones considered in the following is given by

$$
\mathcal{J}(\xi)=\sum_{l=1}^{L} \alpha_{l}\left\|I_{l}^{\mathrm{obs}}-\left|E_{l}^{\mathrm{i}}+\mathbf{K} J_{l}(\xi)\right|^{2}\right\|_{W_{\Gamma}}^{2},
$$

where $I^{\text {obs }}$ represents the available intensity measurements of the total field, and $\alpha_{l}$ is a weighting coefficient set in such a way that the total field intensities corresponding to the different scattering experiments have an equal weight and $W_{\Gamma}$ denotes a weighted $L^{2}$ norm on $\Gamma$. In particular, $\alpha_{l}^{-1}=\left\|I_{l}^{\text {obs }}\right\|_{W_{\Gamma}}^{2}$ and a weighted $L^{2}$ norm, rather than the more usual un-weighted one, is used because of the fact that the adopted cost functional Eq.(9) embeds the solution of a phase retrieval problem for the total field. In these problems, the zeros (or nearly zeros) of the data pattern (in our case $I_{l}^{\text {obs }}$, for each illumination) play a key role in the faithful estimation of the unknown [14], and suggest that a different weight can be herein usefully exploited. Accordingly, we choose a weighting function which emphasizes the contributions to cost functional corresponding to small amplitude data [14]. In particular, the weighing function $w_{l}(\mathbf{r} \in \Gamma)$ is given by

$$
w_{l}(\mathbf{r} \in \Gamma)=\frac{1}{I_{l}^{\mathrm{obs}}(\mathbf{r} \in \Gamma)+\varepsilon}
$$

wherein the positive regularization parameter $\varepsilon$ allows to manage the exact zeros in the data [14]. 
The minimization of $\mathcal{J}$ under the constraints of Eq.(6) can be cast as the global minimization of the following cost functional

$$
\mathcal{L}(\xi, J)=\sum_{l=1}^{L}\left\{\alpha_{l}\left\|I_{l}^{\mathrm{obs}}-\left|E_{l}^{\mathrm{i}}+\mathbf{K} J_{l}(\xi)\right|^{2}\right\|_{W_{\Gamma}}^{2}+\beta_{l}\left\|J_{l}-\xi E_{l}^{\mathrm{i}}-\xi \mathbf{G}_{\bmod }\left(J_{l}\right)\right\|_{D}^{2}\right\}
$$

where $\beta_{l}$ are appropriate weighting coefficients, taken here as $\beta_{l}^{-1}=\left\|E_{l}^{\mathrm{i}}\right\|_{D}^{2},\|\cdot\|_{D}^{2}$ being the un-weighted $L^{2}$ norm over $D$. The gradients of the cost functional, which are derived according to the general strategy outlined in [22], are given by

$$
\begin{aligned}
\nabla_{\xi} \mathcal{L} & =-2 \sum_{l=1}^{L} \beta_{l}\left[E_{l}^{\mathrm{i}}+\mathbf{G}_{\text {mod }}\left(J_{l}\right)\right]^{*}\left[J_{l}-\xi E_{l}^{\mathrm{i}}-\xi \mathbf{G}_{\text {mod }}\left(J_{l}\right)\right] \\
\nabla_{J_{l}} \mathcal{L} & =4 \alpha_{l} \mathbf{K}^{\dagger}\left[\left(E_{l}^{\mathrm{i}}+\mathbf{K} J_{l}\right)\left(I^{\mathrm{obs}}-\left|E_{l}^{\mathrm{i}}+\mathbf{K} J_{l}\right|^{2}\right) w_{l}\right]+ \\
& +2 \beta_{l}\left[\mathbf{I}-\xi \mathbf{G}_{\mathrm{mod}}\right]^{\dagger}\left[J_{l}-\xi E_{l}^{\mathrm{i}}-\xi \mathbf{G}_{\text {mod }}\left(J_{l}\right)\right]
\end{aligned}
$$

where ${ }^{\dagger}$ stands for the transpose conjugate operation, ${ }^{*}$ for the conjugate operation.

A standard minimization gradient-based scheme can now be employed to obtain an estimation of the dielectric properties of the scatterer. Of course, additional a priori information (e.g. positiveness, lossless nature of the targets, ...) can be also considered during the iterative process.

\section{Choice of the starting guess}

An important point in the minimization of Eq.(11) is the choice of the starting guess, i.e. the initial distribution of the auxiliary function and of the contrast source inside the scattering domain. Different solutions can be found in the literature.

A very popular one is the background solution, which consists in choosing an initial contrast function in $D$ slightly different from zero. Then, the corresponding auxiliary function $\xi$ is determined according to Eq.(5), while the contrast source is evaluated by solving Eq.(4). Of course, such a choice, by neglecting the presence of the target in the initial step, does not contain any a priori information.

A second possible choice, more useful and widely used in the framework of the source type integral equation based inversion methods, is the backpropagation solution [18]. In its original formulation, the initial contrast source is first retrieved from the scattered field, which is assumed to be known in both amplitude and phase. Then the contrast function, and thus the auxiliary function in the framework of the CS-EB inversion method, are determined 
by solving Eq.(4) in terms of the contrast function, using the contrast source distribution previously determined.

Of course, this kind of strategy cannot be applied in the present framework, due to the lack of information on the phase distribution. Therefore, a modified version of the above backpropagation solution has been derived. The key idea is to assume an initial distribution of the contrast source in $D$ given by the incident field (which is known or estimated [17]) times a constant given, for instance, by the average value of the auxiliary function in $D$ (or of the contrast function in the framework of the CS inversion method [18]). Then, in order to derive a suitable distribution of the initial auxiliary function and thus of the contrast function, Eq.(4) is solved in terms of the auxiliary function, according to the original strategy. The achieved result is denoted in the following as the modified backpropagation solution. In particular, once the contrast source distribution has been initialized as follows

$$
J_{l, 0}(\mathbf{r})=\gamma E_{l}^{\mathrm{i}}(\mathbf{r})
$$

wherein $\gamma$ is a real constant to determine according to the availability of some information on the nature of the targets (lossless nature, approximate mean value of the permittivity distribution,...), the initial distribution of the auxiliary function is obtained by minimizing the following cost functional

$$
\xi_{0}(\mathbf{r})=\min _{a_{p}}\left\|J_{l, 0}-\xi E_{l}^{\mathrm{i}}-\xi \mathbf{G}_{\bmod }\left(J_{l, 0}\right)\right\|_{D}^{2} .
$$

wherein $\left\{a_{p}\right\}_{p=1}^{P}$ are the auxiliary function coefficients as defined in Eq.(7). As shown in the following, this simple modification of the original backpropagation solution allows to improve the final reconstructions. Note that, in that case, no a priori information on the nature of the target has been considered, except for an approximate knowledge of the contrast average over $D$. Of course, if more information on the objects are available, such as their lossless nature or some positivity constraints on the real part of the permittivity distribution, one can improve the quality of the starting guess and therefore the final results.

\section{Features and limitations of the two-steps approach: a brief overview}

As recalled in the introduction, the problem of reconstructing the unknown contrast from amplitude-only measurements of the total field has been previously approached from a different perspective. The devised method, conceptually alternative to direct ones, such as the 
one presented in Section 3, envisages a solution procedure which splits the imaging problem into two steps.

In the first step, a preliminary estimation of the amplitude and phase patterns of the scattered fields (i.e., the usual input of an inverse scattering approach) is performed solving a quadratic nonlinear inverse problem [1, 13, 17]. To this aim, one uses the fact that the overall complex scattered fields pattern can be represented by means of a set of $P$ Fourier harmonics coefficients on the measurement circle, say $\mathbf{f}=\left\{f_{1}, \ldots, f_{P}\right\}$. Then, one has to minimize the following quantity

$$
\mathcal{F}(\mathbf{f})=\sum_{l=1}^{L}\left\|I_{l}^{\mathrm{obs}}-\left|E_{l}^{\mathrm{i}}\right|^{2}-\left|E_{l}^{\mathrm{s}}\right|^{2}-2 \Re e\left(E_{l}^{\mathrm{i}} E_{l}^{\mathrm{s} *}\right)\right\|_{W_{\Gamma}}^{2} .
$$

For this class of inverse problems, it is known that occurrence of false solutions can be avoided, provided that the ratio among the number of independent data (here the samples of $I^{\text {obs }}$ ) and the number of real unknowns (here given by the real and imaginary parts of f) is larger than 3 [14]. By addressing the reader to [1] for a more detailed discussion, it is worth recalling here that the interference term in the available data between the incident and scattered fields can be conveniently exploited in this framework to fulfil the above mentioned condition and therefore achieve a robust estimate of the (complex) scattered field pattern. In particular, it turns out that, given the size of the minimum circle $R_{D}$ which encloses the targets, a proper setting of the measurement set-up parameters (i.e. the radius $R_{\Gamma}$ of the circumference wherein the probes are located) is sufficient to match the desired ratio. For the geometry at hand (see Fig. 1), the field properties and representations recalled in Section 2 simply leads to $R_{\Gamma}>R_{c r}=(-1+\sqrt{6}) R_{D}$, which thus rules the proper choice of $R_{\Gamma}$ [1]. Of course, when this condition cannot be realized (for instance due to some physical constraints on the set-up dimensions) the solution of the quadratic inverse problem may result in an unreliable estimate of the sought pattern $[1,17]$. It is worth noticing that similar rules can be derived also in the case of aspect limited data [13], by properly taking into account the different geometry.

The second part of the two-steps approach consists in the solution of a traditional inverse scattering problem and can be therefore pursued by taking advantage of any of the many methods developed in the literature so far. For the sake of comparison, we will consider the same CS-EB scattering model as in Section 3. By denoting with $E_{l}^{e s t}$ the estimated scattered field (amplitude and phase), the cost functional to be minimized is now given by 
[20]:

$$
\mathcal{L}(\xi, J)=\sum_{l=1}^{L}\left\{\hat{\alpha}_{l}\left\|\mathbf{K} J_{l}-E_{l}^{e s t}\right\|_{\Gamma}^{2}+\beta_{l}\left\|J_{l}-\xi E_{l}^{\mathrm{i}}-\xi \mathbf{G}_{\bmod }\left(J_{l}\right)\right\|_{D}^{2}\right\},
$$

where, different from the one-step approach and according to its usual definition [18, 20], ${\hat{\alpha_{l}}}^{-1}=\left\|E_{l}^{e s t}\right\|_{\Gamma}^{2}$ and $\beta_{l}$ is the same as in Eq.(11).

By comparing the first term of 17 to the corresponding one in the single-step cost functional of Eq.(11), one can immediately notice that the two-steps approach is characterized by a lower degree of non-linearity [19] with respect to the parameters embedding the unknowns of the inverse problem. In particular, while the first term in Eq.(17) depends on the unknown contrast source $J_{l}$ in a linear way, the corresponding term in Eq.(11) is related to its square amplitude, thus passing from a linear dependence to a non-linear one. Due to the local nature of the adopted optimization method, such a circumstance has a key role in obtaining accurate reconstructions of the unknown permittivity maps [22]. In particular, the single-step approach results to be more sensitive to the starting guess. Accordingly, when the conditions on the measurement set-up makes it applicable, the two step phaseless imaging method has to be preferred.

\section{Experimental results}

The performances of the two phaseless imaging approaches described in Section 3 and Section 4 have been tested using the experimental data provided by the Institute Fresnel of Marseille [23-25]. In these experiments, measurements are collected under an aspect limited configuration in which, for each position of the primary source $\vartheta_{l} \in\left[0,360^{0}\right]$, measurements are gathered over an open arc $\vartheta_{r} \in\left[\vartheta_{l}+60^{0}, \vartheta_{l}+300^{0}\right]$. For all the considered examples, the working frequency is $4 \mathrm{GHz}$. The domain under test is taken as a square region of $2.8 \lambda$ side, $\lambda$ being the wavelenght in free space, subdivided in $46 \times 46$ pixels for the first two examples and in $80 \times 80$ in the last one, according to the metallic nature of the target. As the overall number of scattering experiments ( $L$-number of sources and $M$-number of receivers) are being dependent from the considered experiments, they will be given for each case. The iterative procedure is stopped when the difference between the previous value of the adopted cost functional and the actual one is less than $1.0 \times 10^{-4}$. Moreover, note that when the modified backpropagation solution is used as suitable stating guess, the parameter 
$\gamma$ in Eq.(14) has been fixed equal to 1.0 in all the numerical experiments.

As the database provides measurements of amplitude and phase of the total and incident fields, we can conveniently exploit the measured phase of the total field to check the accuracy of both phaseless imaging approaches herein presented.

\section{A. TwinDielTM dataset}

As first example, we have considered the TwinDiELTM dataset [23] consisting of two dielectric homogeneous cylinders of radius $1.5 \mathrm{~cm}$ and permittivity $3 \pm 0.3$, approximately positioned at $(-4.5,0) \mathrm{cm}$ and $(4.5,0) \mathrm{cm}$, respectively. This dataset is known in the literature as a benchmark for non-linear inverse scattering methods, as linearized approaches are understood to fail when applied to it. In this experiment, $L=36$ source positions and $M=72$ receiver positions have been collected. According to the above results on the field properties, both the intensity of the total field and of the incident field are properly sampled in this example.

Let us start to apply the two-step procedure of Section 4. In particular, by using the knowledge of the incident field and of the measured square amplitude distribution of the total field on the $240^{\circ}$ arc, the Fourier harmonics coefficients of the scattered field are evaluated by minimizing Eq. $(16)$. The number of coefficients (here $11 \times 11$ ) is related to the electrical dimension of the investigated domain and a random distribution has been used as a starting guess in the quadratic minimization procedure. By comparing the actual scattered field (see Fig. 2 and Fig. 3) and the retrieved one (see Fig. 4 and Fig. 5), it can be observed that a good reconstruction is achieved, both in amplitude and in phase, although a slightly worse reconstruction is obtained at the end of the observation arc, due to the truncation of the measurement domain. It is also interesting to note that since the radius of circle wherein the receivers are located is $R_{\Gamma}=1.765 \mathrm{~m}$ and $R_{c r}=0.2113 \mathrm{~m}$, the condition $R_{\Gamma}>R_{c r}$ holds true and then prevents local minima occurence [1].

The second and final step of the two-step based procedure deals with a standard inverse scattering problem, addressed here using the CS-EB method. The background solution has been used as a starting guess in the minimization of Eq.( 17). After representing the unknown auxiliary function $\xi$ and then the contrast function $J$ in terms of $11 \times 11$ Fourier harmonics in the minimization procedure, the very accurate reconstruction of the real part 
of the contrast is achieved and shown in Fig. 6. Note that no a priori information has been used at all. The corresponding imaginary part, in agreement with the lossless nature of the targets, is negligible with respect to the real one $(0.10$ is the maximum value of the estimated imaginary part). The maximum value of the estimated contrast function is 2.1, which is within the measurement accuracy. The overall computational time is about few minutes on a standard Desktop PC.

It is worth noticing that a comparable reconstruction is actually obtained when using the measured (amplitude and phase) scattered fields directly inside Eq.( 17), thus confirming the possibility of performing a faithful phaseless quantitative imaging without any loss of accuracy.

Let us now compare the above result with the one obtained by the one-step approach of Section 3, where the intensity data is directly incorporated into the minimization process. The auxiliary function $\xi$ and the contrast function are still represented in terms of $11 \times 11$ Fourier harmonics. The background solution is first used as a starting guess and the obtained results are represented in Fig. 7. Due to the discussed higher degree of nonlinearity of the one-step inversion problem, the minimization procedure is more sensitive to the starting guess and get stuck into a local minimum. Indeed, even if the shape of the two targets is clearly defined, their permittivity value is much lower than the actual one.

In order to improve the results, the modified backpropagation solution is now used as a starting guess. As shown in Fig. 8, the use of this different initialization leads to an improved reconstruction for the real part of the contrast. Again, the corresponding imaginary part is negligible with respect to the real one, being 0.12 its maximum estimated value.

\section{B. FoamDielExtTM dataset}

As second example, we have considered the FOAMDiELExTTM dataset [24, 25] which corresponds to an inhomogeneous target embedded in free space. The target is made of two purely dielectric cylinders, tangent to each other. The larger one is centered and presents a relative permittivity of $1.45 \pm 0.1$ and a radius of $0.04 \mathrm{~m}$, the smaller one has a relative permittivity of $3 \pm 0.3$ and a radius of $0.015 \mathrm{~m}$. In this case, a combination of $L=8$ source positions and $M=241$ receiver positions are collected. Due to the very limited number of source positions in this example, both the intensity of the total field and of the incident 
field are not properly sampled (see Section 2). Therefore, worse results are expected with respect to previous example, both in terms of reconstruction of the scattered field and of the permittivity profile.

Let us consider first the two-step based procedure. After modeling the unknown complex scattered field as a superposition of $11 \times 11$ Fourier harmonics, we have solved the problem of Eq.( 16); then the unknown auxiliary function and the contrast function have been represented in terms of $11 \times 11$ Fourier harmonics and the cost functional in Eq.( 17) has been minimized starting from the background solution as a suitable starting guess. The real part of the reconstructed contrast is shown in Fig. 9, while the imaginary one, according to the lossless nature of the targets, is again negligible.

It is interesting to note that, as expected, the quality of the reconstruction is worse than the previous example, even if the presence of two targets is clearly retrieved, together with their size and shape. In particular, the reduced number of independent data at our disposal for the inversion of the quadratic operator in the first step (we passed from the 36 sources of the first example to the 8 of this one) makes it more difficult to extract the scattered field from the intensity only of the total field, thus negatively affecting the accuracy of the final reconstruction of the contrast function in the second step.

Let us now consider the one-step procedure. The unknown auxiliary function and the contrast function are represented in terms of $11 \times 11$ Fourier harmonics. By using the modified backpropagation solution as starting guess, the real part of the contrast function reported in Fig. 10 is achieved, whose corresponding imaginary part is negligible (0.07 is now its maximum value).

As it can be seen, by using an accurate starting guess, the final quality of the reconstruction is accurate, both in terms of size of the targets and their shapes, even if the contrast values are slightly underestimated with respect to the actual ones, in agreement with the reduction of independent information. It is also interesting to note that the achieved results are better than the ones obtained by using the two-steps approach of Section 4, thus confirming the expected complementarity and capabilities of the inverse profiling approaches herein discussed. In particular, this example shows that, when the conditions for applying the two-steps procedure do not hold, the one-step approach is a valuable alternative. 


\section{FonMMETExTTM dataset}

To show the capability of the one-step procedure to successfully retrieve the imaginary part of the unknown contrast, which is present in the case of lossy or metallic targets, we have considered the FOAMMETEXTTM dataset [24, 25] which consists of an inhomogeneous target made of two circular cylinders. The smaller one, located outside the dielectric one, is metallic, while the dielectric cylinder is characterized by a relative permittivity of $1.45 \pm 0.1$ and a radius of $0.04 \mathrm{~m}$. The radius of the metallic cylinder is $0.015 \mathrm{~m}$. In this case, $L=18$ and $M=241$ measures are collected.

By still considering $11 \times 11$ unknowns Fourier harmonics for the auxiliary function and the contrast function and by using the modified backpropagation solution as starting guess, the results reported in Fig. 11 and Fig. 12 have been achieved. As it can be seen, the metallic nature of the smaller cylinder has been clearly estimated, as well as its size and shape. We can thus conclude that the procedure can indeed reconstruct the imaginary part of the contrast function. It is also worth noting that the characteristics of the dielectric cylinder have been estimated as well, even if the strong reflection from the metallic target does not allow to achieve an accurate estimation of its shape and size.

Note that the capability to image lossy or metallic targets by means of the two-steps approach has been already investigated and proved, under proper conditions, in [1, 13].

\section{Conclusion}

In this paper two different strategies for the characterization of two-dimensional targets using phaseless measurements of the total fields have been compared by using experimental data measured at the Institute Fresnel of Marseille, concerning homogeneous and inhomogeneous targets, with a combination of purely dielectric and metallic materials.

In the first inversion method, the intensity-only measures of the total field have been directly incorporated in the minimization scheme, while the accuracy and the quality of the final results have been improved by means of a proper definition of the starting guess and a suitable weighting of the considered cost functional.

In the second scheme, originally proposed in [1], one exploits the properties of the scattered fields and the theoretical results on the inversion of quadratic operators to derive a two-steps solution strategy, wherein the (complex) scattered fields embedded in the available data are 
retrieved first and then a traditional inverse scattering problem is solved.

In both cases, the analytical properties and representations of the involved fields allow to properly fix the measurement set-up and to identify the more convenient solution strategy to adopt. In particular, as discussed by means of theoretical considerations and supported by experiments, the two-step strategy achieves better results as compared to the one-step approach, due to its better control of the overall non-linearity of the inverse problem. On the other hand, when the measurement setup does not provide enough independent data to accurately pursue the field retrieval, the one-step approach has to be preferred.

It is interesting to remark that, while the Marseille data are usually elaborated by using multi-frequency (i.e., broad-band) data, the accurate results achieved herein with both approaches only rely on monochromatic data. Of course, use of multi-frequency information can further improve the final results in terms of size, shape estimation and permittivity value.

As further comment, note that in order to have a completely phaseless imaging method the knowledge of the incident field, both in amplitude and in phase, can also be avoided. In particular an additional step would be required in order to estimate the phase of the incident field on the measurement domain before using one of the imaging procedures discussed in this paper. For more details, the reader is addressed to [17].

As future work, note that the development of new and effective inversion approaches starting from only intensity data of the scattered field instead of the total one can be pursued. As a matter of fact, this situation is of interest in optical applications where the capability of imaging dielectric and metallic targets and developing inversion strategies based on phaseless measures can open the way to very interesting applications. On the other side, such a problem sets new challenging difficulties. As a matter of fact, the amount of independent data which can be exploited is considerably reduced both because one cannot exploit interference amongst the incident and the scattered fields (see Sect.4), and because of the fact that the actual measurement set-ups produce aspect limited data [3, 4]. As a consequence, in order to compensate for the lack of information, both multi-frequency approach to the problem and/or the development of innovative measurement configurations (as well as new inversion procedures) are needed. 


\section{Acknowledgement}

This work has been partially supported by the GALILEO programme and by the CNR/CNRS action for the Scientific Exchange between Italy and France.

\section{Appendix A}

Aim of this Appendix is to briefly derive the CS-EB scattering equation [20], adopted in the optimization problems considered in this paper. The starting point is the traditional Contrast - Source integral equation (3); by adding and subtracting the contrast source function $J(\mathbf{r})$ in the integral term in this latter, one gets

$$
J(\mathbf{r})-\chi(\mathbf{r}) E^{\mathrm{i}}(\mathbf{r})=\chi(\mathbf{r}) \iint_{D} G\left(\mathbf{r}, \mathbf{r}^{\prime}\right)\left[J\left(\mathbf{r}^{\prime}\right)-J(\mathbf{r})\right] \mathrm{d} \mathbf{r}^{\prime}+\chi(\mathbf{r}) J(\mathbf{r}) \iint_{D} G\left(\mathbf{r}, \mathbf{r}^{\prime}\right) \mathrm{d} \mathbf{r}^{\prime} .
$$

Then, by grouping with respect to $J(\mathbf{r})$, one achieves

$$
J(\mathbf{r})-\chi(\mathbf{r}) J(\mathbf{r}) f_{D}(\mathbf{r})=\chi(\mathbf{r}) E^{\mathrm{i}}(\mathbf{r})+\chi(\mathbf{r}) \iint_{D} G\left(\mathbf{r}, \mathbf{r}^{\prime}\right)\left[J\left(\mathbf{r}^{\prime}\right)-J(\mathbf{r})\right] \mathrm{d} \mathbf{r}^{\prime}
$$

where

$$
f_{D}(\mathbf{r})=\iint_{D} G\left(\mathbf{r}, \mathbf{r}^{\prime}\right) \mathrm{d} \mathbf{r}^{\prime}
$$

Now, by introducing

$$
\begin{aligned}
\xi(\mathbf{r}) & =\frac{\chi(\mathbf{r})}{1-\chi(\mathbf{r}) f_{D}(\mathbf{r})} \\
\mathbf{G}_{\bmod }(J) & =\iint_{D} G\left(\mathbf{r}, \mathbf{r}^{\prime}\right)\left[J\left(\mathbf{r}^{\prime}\right)-J(\mathbf{r})\right] \mathrm{d} \mathbf{r}^{\prime}=\iint_{D} G\left(\mathbf{r}, \mathbf{r}^{\prime}\right) J\left(\mathbf{r}^{\prime}\right) \mathrm{d} \mathbf{r}^{\prime}-J(\mathbf{r}) f_{D}(\mathbf{r}) .
\end{aligned}
$$

and replacing them into Eq.(A.2), the CS-EB scattering equation (4) is achieved [20].

Some comments are now in order. First, note that the CS-EB equation has been derived without any approximation from the CS one, being thus completely equivalent to it. Moreover, since the two equations have the same structure, one can exploit all numerical algorithms and tools already developed for the CS equation for the solution of the CS-EB one. On the other hand, a new radiation operator and a different auxiliary function, see Eq.(A.4), are involved in the CS-EB equation. Therefore, as detailed in [20], Eq.(3) and Eq.(4) have the same information content, but the CS-EB equation exhibits a different, actually lower, degree of non linearity [19] of the relationship amongst parameters embedding dielectric characteristics and the scattered fields. As such, it defines a new and convenient model for solving forward and inverse scattering problems [20]. 


\section{References}

1. L. Crocco, M. D’Urso and T. Isernia, "Inverse scattering from phaseless measurements of the total field on a closed curve", J.Opt. Soc. Am. A, 21, 622-631 (2004).

2. D. Colton and R. Kress, "Inverse Acoustic and Electromagnetic Scattering Theory", Springer Verlag Series in Applied Mathematics, 93 (1998).

3. P. C. Chaumet, K. Belkebir and A. Sentenac, "Superresolution of three-dimensional optical imaging by use of evanescent waves", Opt. Letters, 29, 2740-2742 (2004).

4. P. C. Chaumet, K. Belkebir, R. Lencrerot, "Three-dimensional optical imaging in layered media", Opt. Express, 14, 3415-3426 (2006).

5. M. A. Fiddy, M. Testorf, "Inverse scattering method applied to the synthesis of strongly scattering structures", Opt. Express, 14, 2037-2046 (2006).

6. V. Lauer, "New approach to optical diffraction tomography yielding a vector equation of diffraction tomography and a novel tomographic microscope", J. Microsc., 205, 165176 (2002).

7. N. Destouches, C.A. Guérin, M. Lequime and H. Giovannini, "Determination of the phase of the diffracted field in the optical domain. Application to the reconstruction of surface profiles", Opt. Commun., 198, 233-239 (2001).

8. M. H. Maleki, A.J. Devaney, A. Schatzberg, "Tomographic reconstruction from optical scattered intensities", J. Opt. Soc. Am. A, 10, 1356-1363 (1992).

9. M.H. Maleki, A.J. Devaney, "Phase retrieval and intensity-only reconstruction algorithms from optical diffraction tomography", J. Opt. Soc. Am. A, 10, 1086-1092 (1993).

10. T. Takenaka, D. J. N. Wall, H. Harada, M. Tanaka, "Reconstruction algorithm of the refractive index of a cylindrical object from the intensity measurements of the total field", Microwave Opt. Technol. Lett., 14, 182-188 (1997).

11. S. Caorsi, A. Massa, M. Pastorino, A. Randazzo, "Electromagnetic detection of dielectric scatterers using phaseless synthetic and real data and the Memetic Algorithm", IEEE Trans. Geosci. Remote Sens., 23, 2745-2752 (2003).

12. M. Benedetti, M. Donelli, A. Massa, "Inversion of phaseless total field data using a two-step strategy based on the iterative multiscaling approach", IEEE Trans. Geosci. Remote Sens., 23, 2745-2752 (2003).

13. O. M. Bucci, L. Crocco, M. D’Urso, T. Isernia, "Inverse scattering from phaseless 
measurements of the total field on open lines", J. Opt. Soc. Am. A, 23, 2566-2577 (2006).

14. T. Isernia, G. Leone, R. Pierri, and F. Soldovieri, "Role of the support and zero locations in phase retrieval by a quadratic approach", J. Opt. Soc. Am. A, 16, 1845-1856 (1999).

15. O.M. Bucci, C. Gennarelli, and C. Savarese, "Representation of electromagnetics fields over arbitrary surfaces by a finite and nonredundant number of samples", IEEE Trans. Antennas Propag., 46, 351-359 (1998).

16. O. M. Bucci, T. Isernia, "Electromagnetic inverse scattering: retrievable information and measurement strategies", Radio Science, 32, 2123-2138 (1997).

17. L. Crocco, M. D'Urso and T. Isernia, "Faithful non-linear imaging from only-amplitude measurements of incident and total fields", Optics Express, 15, 3804-3815 (2007).

18. R. E. Kleinman and P. M. van den Berg, "A Contrast Source inversion method", Inverse Probl., 13, 1607-1620 (1997).

19. O. M. Bucci, N. Cardace, L. Crocco, T. Isernia, "Degree of non-linearity and a new solution procedure in scalar 2-D inverse scattering problems", J. Opt. Soc. Am. A, 18, 1832-1843 (2001).

20. T. Isernia, L. Crocco, and M. D'Urso, "New Tools and Series for Forward and Inverse Scattering Problems in Lossy Media," IEEE Trans. Geosci. Remote Sens. Lett., 1, 327-331 (2004).

21. L. Crocco, M. D’Urso and T. Isernia, "Testing the Contrast Source Extended Born method against real data: the TM case," Inverse Probl., 21, S33-S50 (2005)

22. T. Isernia, V. Pascazio, and R. Pierri, "On the local minima in a tomographic imaging technique", IEEE Trans. Geosci. Remote Sens. Lett., 39, 1596-1607 (2001).

23. K. Belkebir and M. Saillard, "Special section: Testing inversion algorithms against real data", Inverse Probl., 17 (2001).

24. K. Belkebir and M. Saillard, "Special section: Testing inversion algorithms against real data: inhomogeneous targets", Inverse Probl., 21 (2005).

25. J.-M. Geffrin, P. Sabouroux and C. Eyraud, "Free space experimental scattering database continuation: experimental set-up and measurement precision", Inverse Problems, 21, S117-S130 (2005). 


\section{Figure Captions}

- Fig. 1 - Geometry of the problem. A two-dimensional target with cross-section $\Omega$. $\Gamma$ (with radius $b$ ) is the circle where the sources $\left(T_{x}\right)$ and probes $\left(R_{x}\right)$ are located. $a$ is the radius of the minimum circle enclosing the unknown targets.

- Fig. 2 - Measured intensity of the scattered fields for the TwinDiELTM dataset.

- Fig. 3 - Measured phase of the scattered fields for the TwinDiELTM dataset.

- Fig. 4 - Reconstructed intensity of the scattered fields for the TwinDiELTM dataset obtained by solving Eq.(16) of the two-steps procedure in Section 4.

- Fig. 5 - Reconstructed phase of the scattered fields for the TwinDiELTM dataset obtained by solving Eq.(16) of the two-steps procedure in Section 4.

- Fig. 6 - Real part of the reconstructed contrast function for the TwinDielTM dataset when using Eq.(17) of the two-steps procedure in Section 4. The maximum value of the estimated contrast function is 2.10 .

- Fig. 7 - Real part of the reconstructed contrast function for the TwinDiELTM dataset with the one-step approach of Section 3. The background solution has been used as strating guess. The maximum value of the estimated contrast function is 0.67.

- Fig. 8 - Real part of the reconstructed contrast function for the TwinDiELTM dataset obtained with the one-step procedure of Section 3. The modified backpropagation solution has been used as starting guess in the inversion procedure. The maximum value of the estimated contrast function is 1.85 .

- Fig. 9 - Real part of the reconstructed contrast function for the FOAMDIELEXTTM dataset obtained when following the two-steps approach of Section 4. The background solution has been used as starting guess. The maximum value of the estimated contrast function is 1.34 .

- Fig. 10 - Real part of the reconstructed contrast function for the FOAMDiELExTTM dataset by using the one-step approach of Section 3. The modified backpropagation solution has been used as starting guess. The maximum value of the estimated contrast function is 1.75 . 
- Fig. 11 - Real part of the reconstructed contrast function for the FoAmMetExtTM dataset by using the one-step approach of Section 3. The modified backpropagation solution has been used as starting guess.

- Fig. 12 - Imaginary part of the reconstructed contrast function for the FOAMMETExTTM dataset by using the one-step approach of Section 3. The modified backpropagation solution has been used as starting guess. 


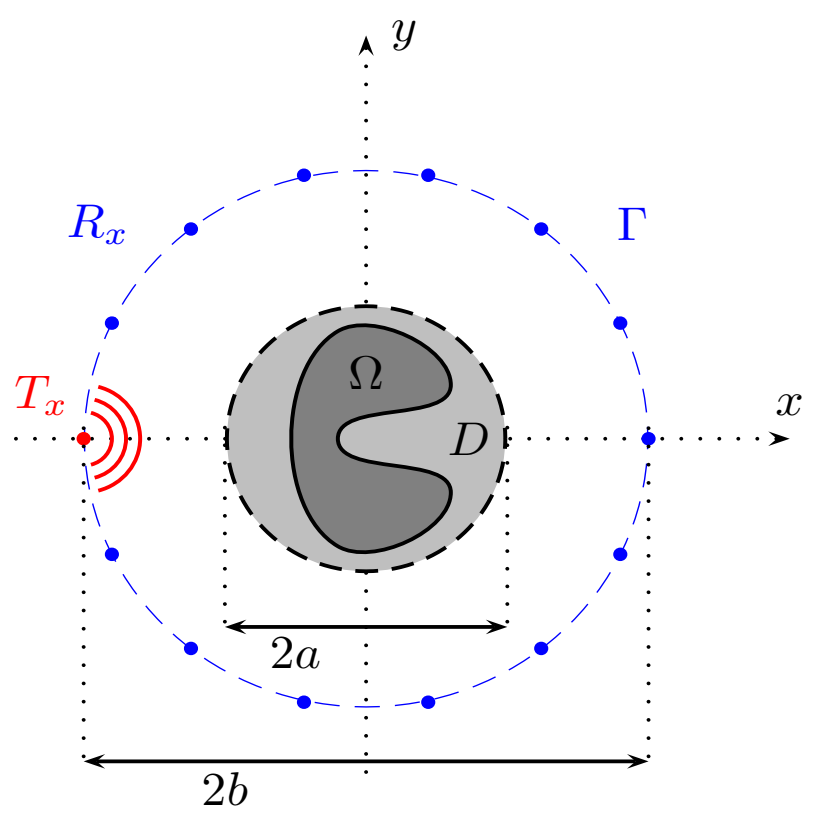

Fig. 1. Geometry of the problem. A two-dimensional target with cross-section $\Omega$. $\Gamma$ (with radius $b)$ is the circle where the sources $\left(T_{x}\right)$ and probes $\left(R_{x}\right)$ are located. $a$ is the radius of the minimum circle enclosing the unknown targets. 


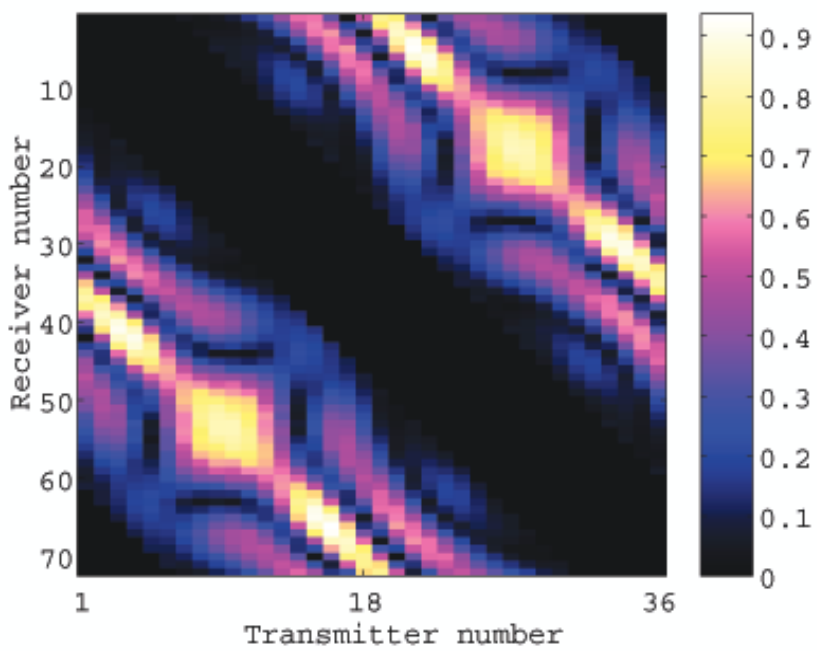

Fig. 2. Measured intensity of the scattered fields for the TwinDiELTM dataset. 


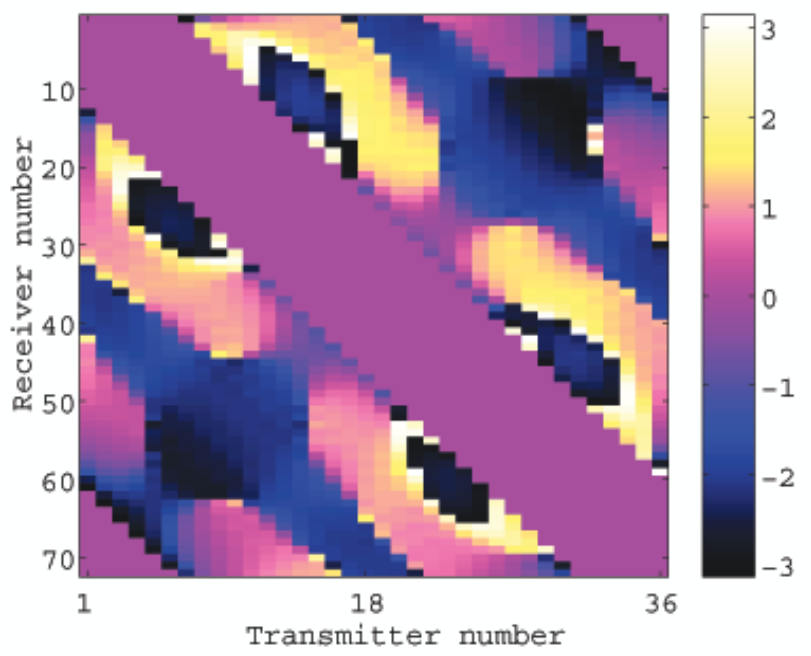

Fig. 3. Measured phase of the scattered fields for the TwinDielTM dataset. 


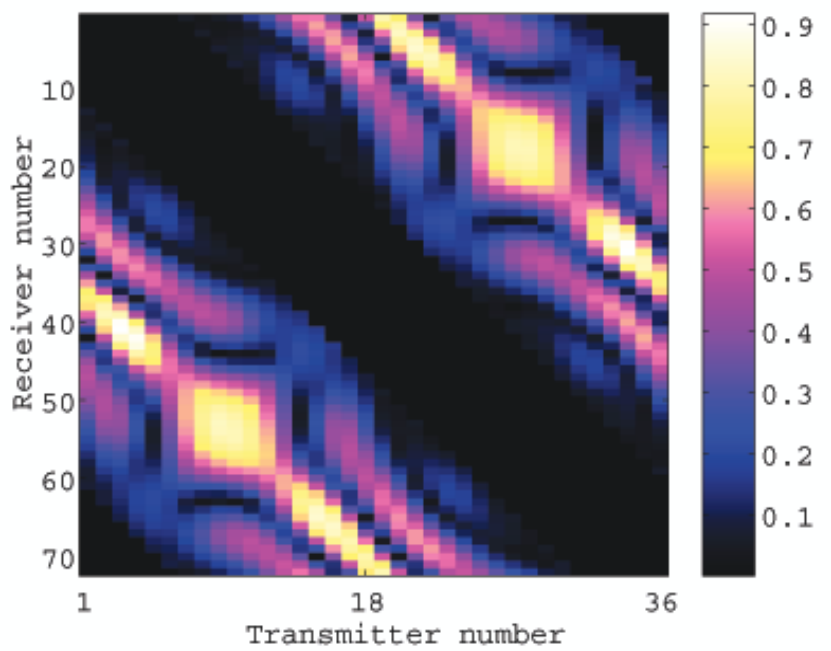

Fig. 4. Reconstructed intensity of the scattered fields for the TwINDiELTM dataset obtained by solving Eq.(16) of the two-steps procedure in Section 4. 


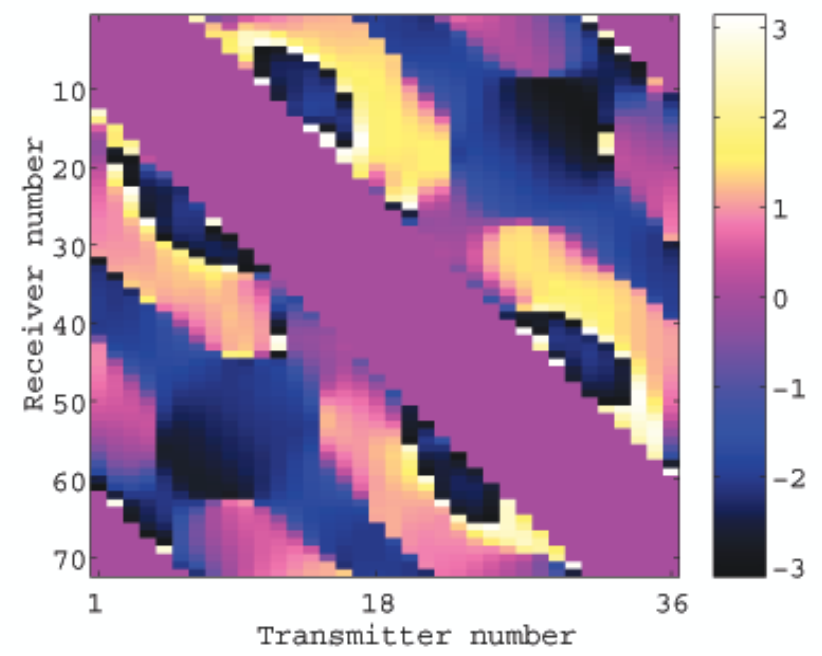

Fig. 5. Reconstructed phase of the scattered fields for the TwINDiELTM dataset obtained by solving Eq.(16) of the two-steps procedure in Section 4. 


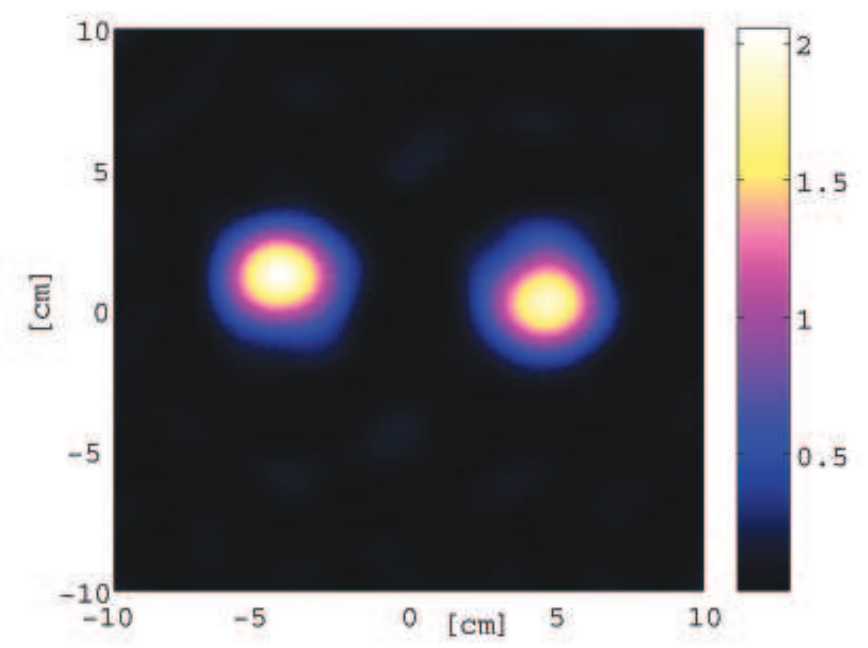

Fig. 6. Real part of the reconstructed contrast function for the TwinDiELTM dataset when using Eq.(17) of the two-steps procedure in Section 4. The maximum value of the estimated contrast function is 2.10 . 


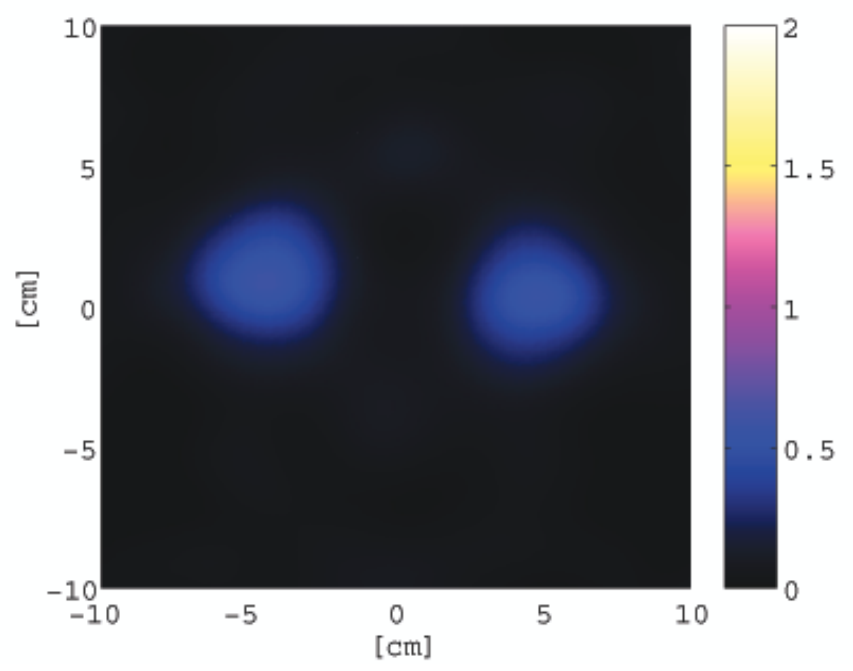

Fig. 7. Real part of the reconstructed contrast function for the TwINDIELTM dataset with the one-step approach of Section 3. The background solution has been used as strating guess. The maximum value of the estimated contrast function is 0.67 . 


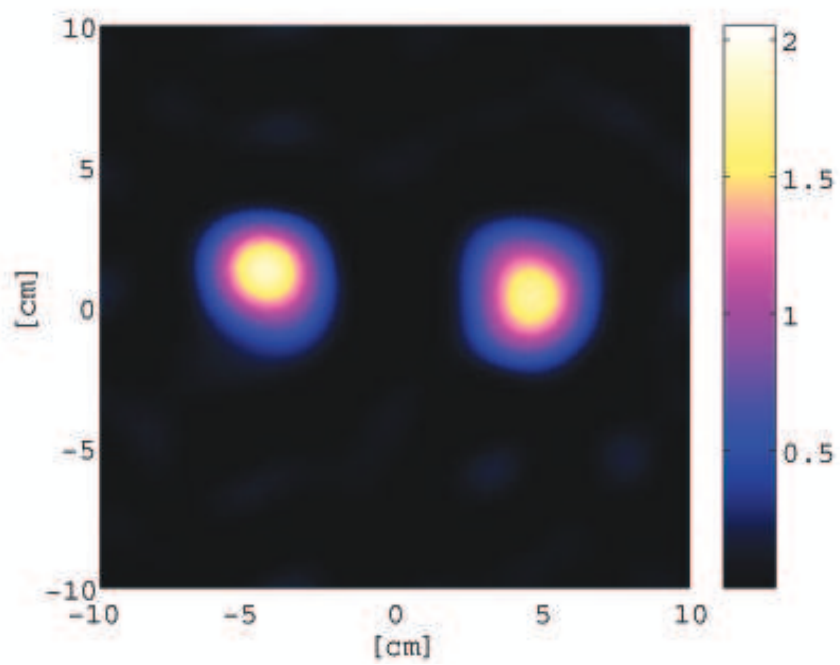

Fig. 8. Real part of the reconstructed contrast function for the TwinDielTM dataset obtained with the one-step procedure of Section 3. The modified backpropagation solution has been used as starting guess in the inversion procedure. The maximum value of the estimated contrast function is 1.85 . 


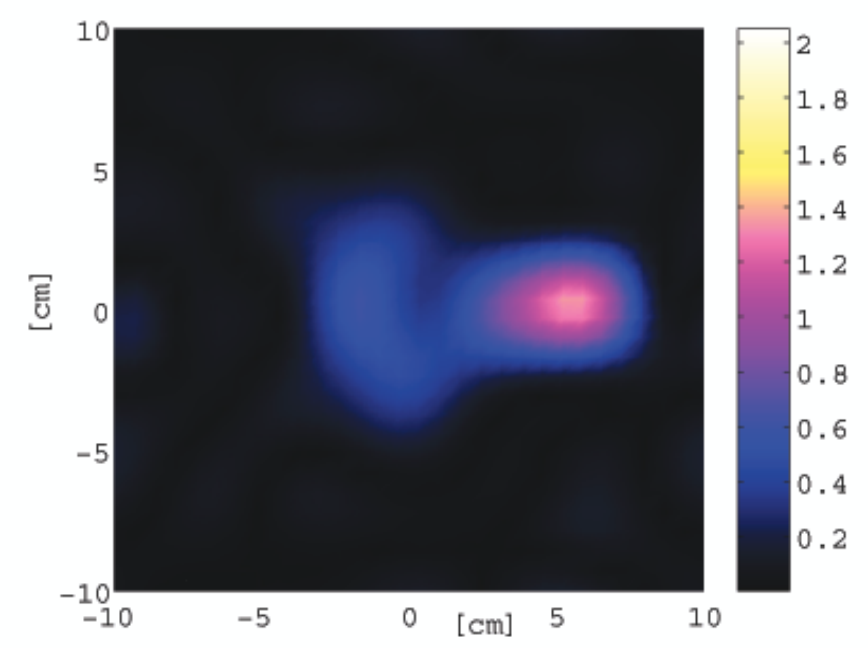

Fig. 9. Real part of the reconstructed contrast function for the FOAMDiELExTTM dataset obtained when following the two-steps approach of Section 4. The background solution has been used as starting guess. The maximum value of the estimated contrast function is 1.34 . 


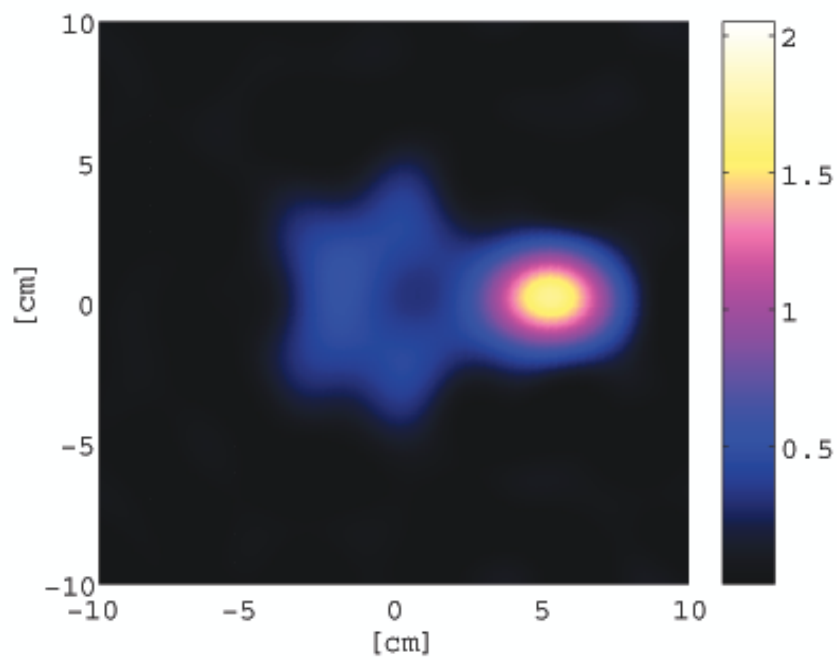

Fig. 10. Real part of the reconstructed contrast function for the FOAMDiELExTTM dataset by using the one-step approach of Section 3. The modified backpropagation solution has been used as starting guess. The maximum value of the estimated contrast function is 1.75 . 


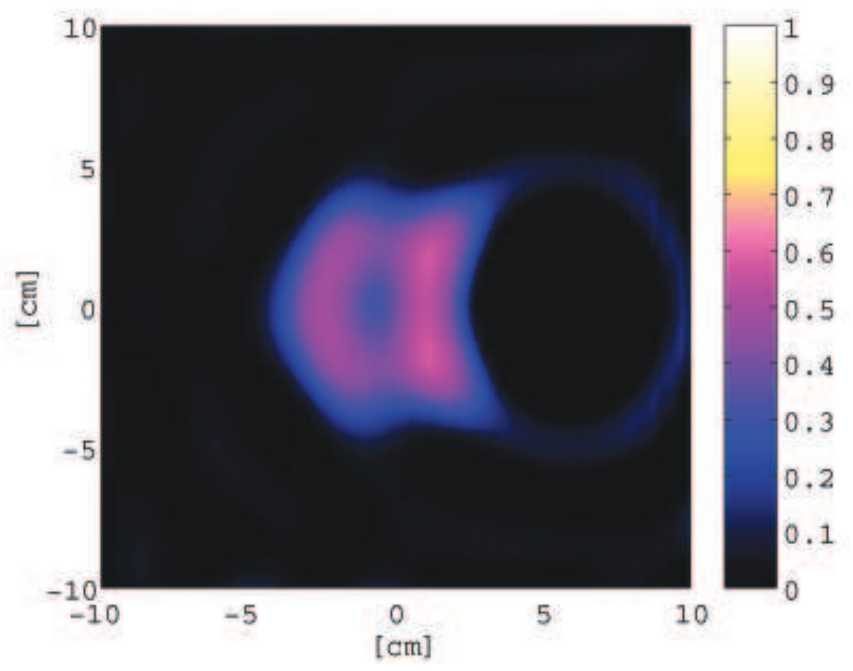

Fig. 11. Real part of the reconstructed contrast function for the FoAmMETExTTM dataset by using the one-step approach of Section 3. The modified backpropagation solution has been used as starting guess. 


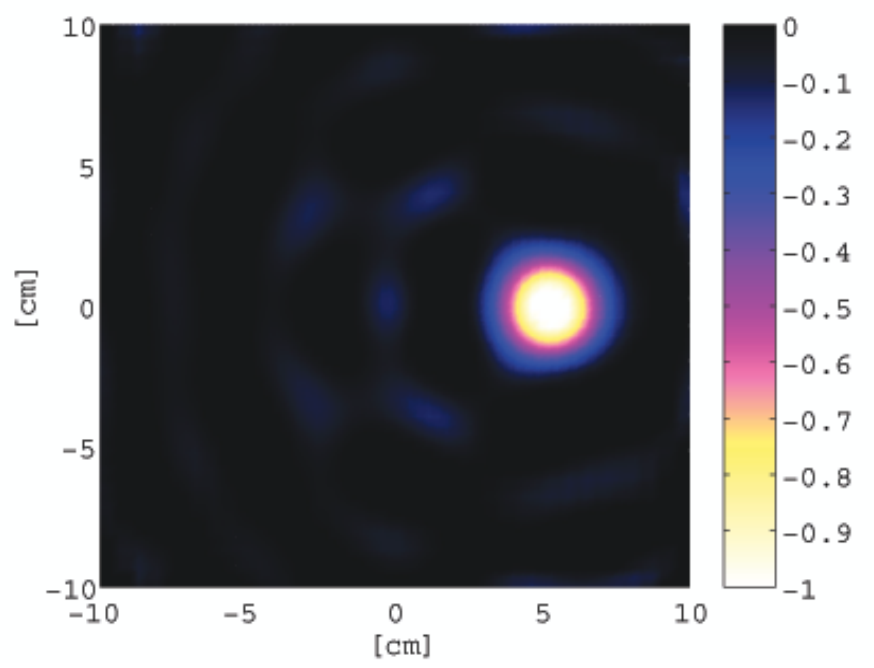

Fig. 12. Imaginary part of the reconstructed contrast function for the FOAMMETEXTTM dataset by using the one-step approach of Section 3. The modified backpropagation solution has been used as starting guess. 